\title{
A traitor in our midst: mesenchymal stem cells contribute to tumor progression and metastasis
}
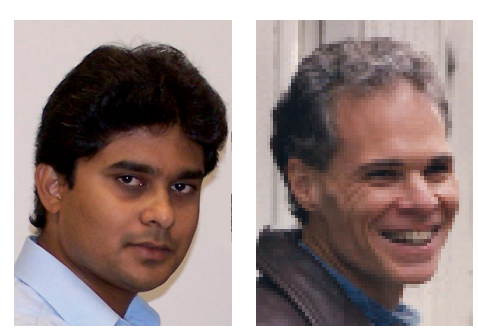

“...just as traitors are punished within society, a therapeutic approach in which traitorous MSCs are functionally isolated or outright executed may ultimately constitute an efficacious treatment for advanced cancer patients."

\section{Prasun J Mishra ${ }^{\dagger}$ \& Glenn Merlino}

†Author for correspondence: Laboratory of Cancer Biology and Genetics, National Cancer Institute, National Institutes of Health, Bethesda, MD 20892-24264, USA = Tel.. +1 3014518522 - Fax: +1 3014807618 =mishrapj@mail.nih.gov

"It is hard to be a traitor, it is a creative act. It requires relinquishing one's identity, losing face.

One must vanish from the picture, remain unrecognized"

- Deleuze G/Parnet C [101].

Although this quote seems appropriate when applied to historical figures, such as Benedict Arnold or Judas, recent reports now provide strong evidence that 'traitorous' can also be used to describe some acts performed by human bonemarrow-derived mesenchymal stem cells (MSCs) $[1,2]$. MSCs are adult multipotent stromal cells with the potential to differentiate into a variety of cell types. They are able to migrate toward an injury site and when there become bone, cartilage, fat or muscle, repairing or replacing the damaged connective tissue; however, MSCs can also be recruited to tumor sites [3-5], perhaps with the best of intentions - to discourage the development of tumors. However, new evidence now demonstrates that MSCs can be persuaded by tumor cells to become phenotype turncoats, morphing into carcinoma-associated fibroblasts that actually support tumorigenesis [1]. Studies that demonstrate that secretions from these MSCs into the tumor microenvironment can then train tumor cells to become more metastatic reveal the full extent of this betrayal [2].

Although studies on bone marrow date back to the 19th century [6], in the last five decades the field has grown tremendously, with approximately 7000 articles listed in PubMed alone. When it was discovered early on that bone marrow cell suspensions had the potential to form individual colonies in vitro, the name 'colony-forming unitsfibroblasts' was suggested [7]. Later, bone marrow cells with nonhematopoietic, plastic-adherent and fibroblast-like properties became known as stromal cells [8-11], osteogenic stem cells [12] or bone marrow stromal stem cells [13]. In 1991, a decade after the isolation of embryonic stem cells, the term 'mesenchymal stem cell' was coined [14], inspired by the ability of single bone marrow stromal cells to differentiate or be re-programmed into a variety of cellular lineages (FIgure 1) [15-18] (reviewed in $[17,18]$ ). It is now well appreciated that MSCs exquisitely influence, and are influenced by, their microenvironment through exposure to specific factors and stimuli [13-18].

What about the possible association between human MSCs re-programming and cancer? Interestingly, some earlier reports in the field had already raised the possibility that the MSC, a master of disguise, can be a precursor for carcinoma- or tumor-associated fibroblasts (CAFs). It was suggested that a subset of CAFs in the tumor microenvironment arise from circulating fibroblasts derived from bone marrow cells, possibly MSCs [19,20]. Certain features of MSCs supported the notion that they could give rise to CAFs, including their potential to differentiate into myofibroblasts, expressing specific markers for the myogenic lineage. Increased expression of $\alpha$-smooth muscle actin was observed in response to TGF- $\beta$, a growth factor often secreted by tumor cells [21,22]. Bone marrow precursor cells were also suggested as a source of the myofibroblasts found at wound healing sites [23].

Recently, it was demonstrated that prolonged exposure to factors in tumor-conditioned medium (TCM) could induce the differentiation of MSCs into a CAF-like morphology (Figure 1) [1]. These TCM-activated MSCs expressed high levels of $\alpha$-smooth muscle actin, vimentin, fibroblast surface protein and stromal

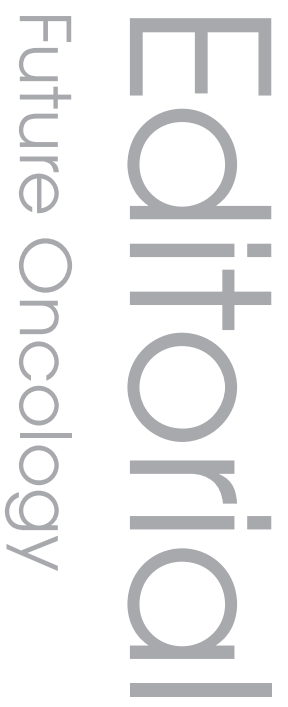

\section{future Mend $_{\text {medicine }}^{\text {fsg }}$}




\section{Editorial Mishra \& Merlino}

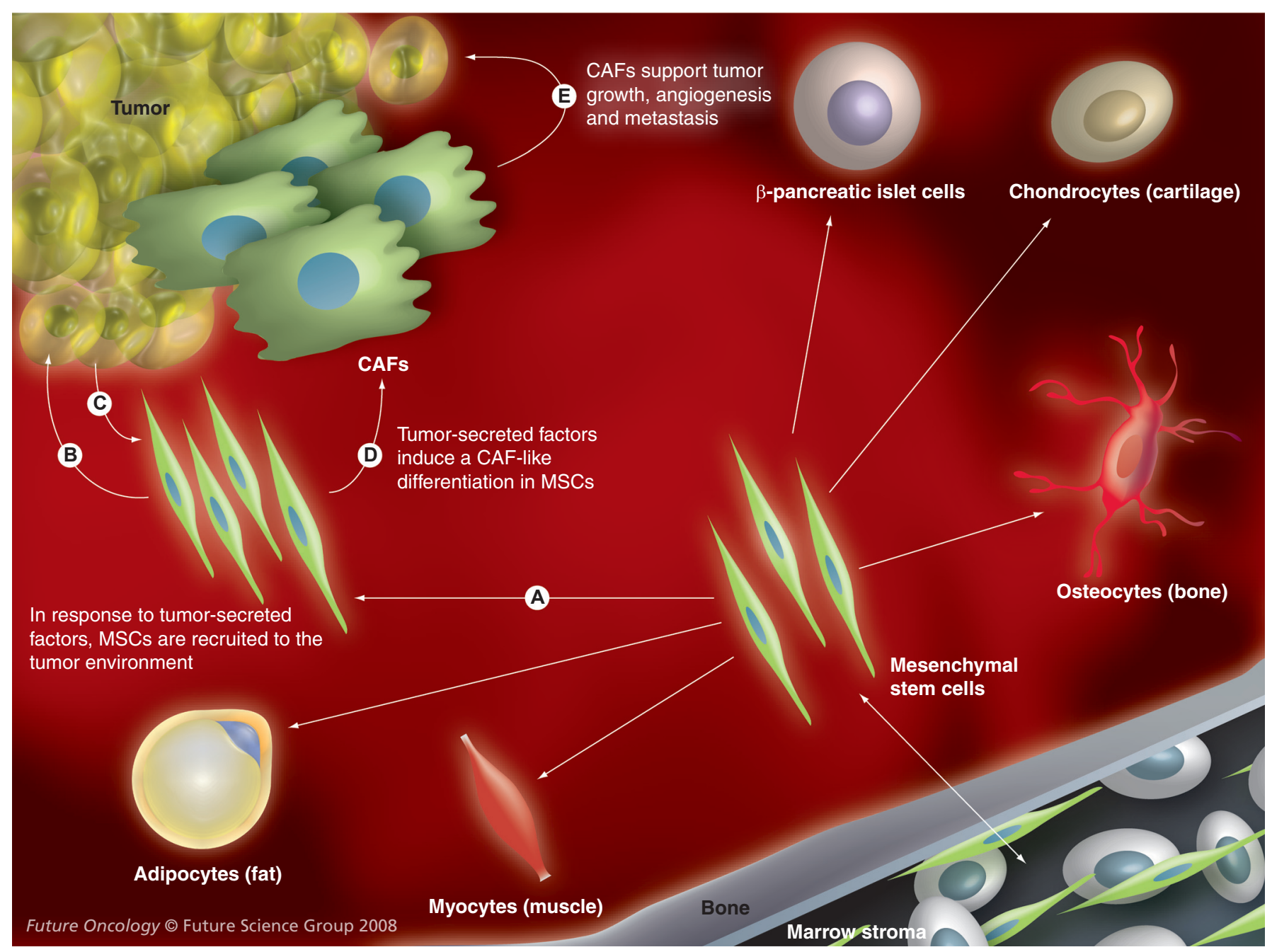

Figure 1. Mesenchymal stem cells support tumor growth and metastasis. MSCs are adult multipotent stem cells with the ability to migrate toward an injury site and repair or replace the damaged connective tissue. Depending on the tissue type, MSCs are able to repair damaged tissues by differentiating into a variety of cell types, such as osteocytes (bone), chondrocytes (cartilage), adipocytes (fat), myocytes (muscle) and $\beta$-pancreatic islet cells. Recently, it was demonstrated that in response to tumor-secreted factors, MSCs are also recruited to tumor sites (A). Once at the tumor site, MSC-secreted factors can have an inhibitory effect on tumor growth (B). However, after long-term exposure to tumor-secreted factors, MSCs can be persuaded by tumor cells (C) to transform into carcinoma-associated fibroblasts able to support tumorigenesis (D). Secretions, such as SDF-1 and CCL5, from tumor-activated MSCs present in the tumor microenvironment can actually train tumor cells to become more tumorigenic and metastatic (E). The ability of MSCs to support the growth and metastatic spread of tumors represents another striking example of the importance of tumor stroma in the development and progression of cancer. CAF: Carcinoma- or tumor-associated fibroblast; CCL5: CC chemokine ligand 5; MSC: Mesenchymal stem cell; SDF-1: Stromal-derived factor 1.

derived factor-1, and morphologically resembled myofibroblasts. These CAF-like MSCs were further compared with MSCs treated with 5-azacytidine, which also triggers MSC differentiation into myofibroblasts [1,15,24-26]. While both expressed myofibroblast lineage markers, MSCs differentiated by 5 -azacytidine and TCM were different in several significant ways. Although microarray-based profiling studies demonstrated that gene-expression patterns in myofibroblasts derived from treatment with either 5-azacytidine or TCM were somewhat similar, only the TCM-activated MSCs were characterized by an upregulation of CAF-associated genes. From a functional standpoint, TCM-derived myofibroblasts could support in vivo tumor growth in nude mice, whereas 5-azacytidine-derived myofibroblasts and naive MSCs could not [1]. Hence, in the tumor microenvironment, MSCs can be induced by specific tumor-derived factors to relinquish their identity and differentiate into CAFs that can support tumor growth. These data indicate that different environmental factors induce morphologically similar, but functionally distinct, alterations in MSCs [1]. Moreover, these studies suggest that tumor-secreted factors possess the ability to recruit MSCs and trigger their differentiation (Figure 1). 
An earlier report by Karnoub et al. demonstrated that subcutaneous coadministration of MSCs with a weakly metastatic human breast carcinoma cell line, MDA-MB-231 (MDA), increased the metastatic potential of that line in a xenograft tumor model [2]. This report also showed that once in contact with tumor cells, MSCs begin to secrete the chemokine CCL5 (also known as RANTES). The CCL5 secreted by the MSCs was able to enhance the motility and metastatic capabilities of MDA breast cancer cells [2]. Moreover, silencing the CCL5-mediated crosstalk between MDA and MSCs reversed the metastatic phenotype. Hence, the phenotypic changes induced by CCL5 in the tumor cells were both reversible and dependent on CCL5 receptor signaling [2]. Examined more broadly, these data raise the novel possibility that the acquisition of metastatic ability by tumor cells may be explained, at least in part, by reversible changes exerted through MSCs recruited to the immediate tumor microenvironment, most likely from the bone marrow, this being the primary source of circulating MSCs. Thus, success at reversing MSC-mediated metastatic training of tumor cells (i.e., by targeting CCL5) may profoundly enhance prospects for cancer prevention and/or therapy [2].

Notably, metastasizing breast cancer cells have been observed to move in association with macrophages [28], raising some interesting questions: since MSCs work in close proximity to tumor cells, do they accompany the tumor cell to its secondary metastatic site? If so, does the MSC continue to influence the tumor cell by maintaining its metastatic re-programming and/or by facilitating colonization through creation of a favorable niche? These questions should be answered in the not-too-distant future.

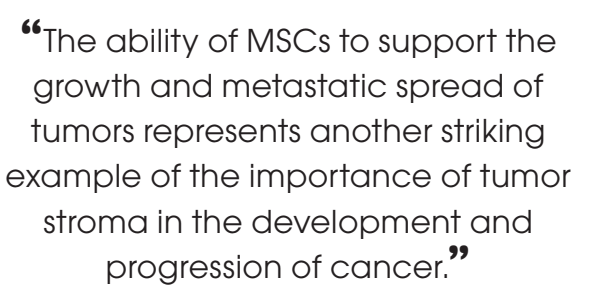

Recently, it has also been demonstrated that epithelial cells can assume a MSC-like morphology by undergoing epithelial-mesenchymal transition (EMT) [29]. EMT is a developmental process in which proliferating epithelial cells become less adherent, more motile and assume a migratory, mesenchymal phenotype [30-33]. Since loss of cell adhesion and enhanced cell mobility is also a characteristic of early metastatic tumor cells, EMT has long been thought to be associated with metastasis [33,34]. Mani et al. have shown that immortalized human mammary epithelial cells can undergo EMT and acquire MSC-like properties, such as an increased ability to form mammospheres [29]. EMT markers were found to be expressed on the stem cells present in the mammary carcinomas. This study also demonstrated that transformed epithelial cells that undergo EMT were more efficient at forming mammospheres, soft agar colonies and tumors compared with control cells [29]. However, the MDA cells that were used in previous studies were in partial EMT and retained distinctive epithelial morphology [2], and when injected subcutaneously without MSCs were unable to support tumor growth [1]. Taken together, these data suggest that MSCs, regardless of their source of origin (e.g., from EMT or bone marrow), harbor the potential to initiate a tumor and/or contribute to its metastatic potential.

66...regardless of their source of origin ...

(MSCs) harbor the potential to initiate a

tumor and/or contribute to its metastatic potential."

It is worth noting that MSCs might also influence tumor progression through interactions with the immune system. In vitro studies have clearly demonstrated that MSCs possess potent immunosuppressive activities affecting both innate and cellular immune pathways [35]. It is not difficult to envision how the interference of T-cell/dendritic cell function by MSC-secreted cytokines could facilitate tumor progression.

These observations suggest that MSCs can support the growth of tumors, and even drive their cells to a metastatic fate, representing another striking example of the importance of tumor stroma in the development and progression of cancer. Although the factors and interactions involved in this process will require further functional characterization, these early experimental findings immediately suggest new therapeutic opportunities for cancer. Such opportunities may be facilitated by the fact that clinical strategies are already being devised in which MSC biology will be exploited for the treatment of various diseases. Therefore, just as traitors are punished within society, a therapeutic approach in which traitorous MSCs are functionally isolated or outright executed may ultimately constitute an efficacious treatment for advanced cancer patients. 


\section{Financial \& competing interests disclosure \\ The authors have no relevant affiliations or financial involvement with any organization or entity with a financial interest in or financial conflict with the subject matter or materials discussed in the manuscript.}

This includes employment, consultancies, honoraria, stock ownership or options, expert testimony, grants or patents received or pending, or royalties.

No writing assistance was utilized in the production of this manuscript.

\section{Bibliography}

Papers of special note have been highlighted as:

- of interest

- of considerable interest

1. Mishra PJ, Humeniuk R, Medina DJ et al.: Carcinoma-associated fibroblast-like differentiation of human mesenchymal stem cells. Cancer Res. 68, 4331-4339 (2008).

- Demonstrated that human bone marrow derived mesenchymal stem cells (MSCs) are a source of carcinoma- or tumor-associated fibroblasts.

2. Karnoub AE, Dash AB, Vo AP et al.: Mesenchymal stem cells within tumour stroma promote breast cancer metastasis. Nature 449, 557-563 (2007).

- Demonstrated that MSCs can support breast cancer metastasis.

3. Studeny M, Marini FC, Champlin RE, Zompetta C, Fidler IJ, Andreeff M: Bone marrow-derived mesenchymal stem cells as vehicles for interferon- $\beta$ delivery into tumors. Cancer Res. 62, 3603-3608 (2002).

4. Studeny M, Marini FC, Dembinski JL et al:: Mesenchymal stem cells: potential precursors for tumor stroma and targeteddelivery vehicles for anticancer agents. J. Natl Cancer Inst. 96, 1593-1603 (2004).

1. Hung SC, Deng WP, Yang WK et al.: Mesenchymal stem cell targeting of microscopic tumors and tumor stroma development monitored by noninvasive in vivo positron emission tomography imaging. Clin Cancer Res. 11, 7749-7756 (2005).

2. Goujon E: Recherches experimentales sur les proprietes physiologiques de la moelle des os. Journal de L'Anat et de La Physiol. 6, 399-412 (1869).

3. Friedenstein AJ, Chailakhjan RK, Lalykina KS: The development of fibroblast colonies in monolayer cultures of guinea-pig bone marrow and spleen cells. Cell Tissue Kinet. 3, 393-403 (1970).

- Landmark paper that identified MSCs and called them colony forming units.

4. Friedenstein AJ, Chailakhyan RK, Latsinik NV, Panasyuk AF, Keiliss-Borok IV: Stromal cells responsible for transferring the microenvironment of the hemopoietic tissues. Cloning in vitro and retransplantation in vivo. Transplantation 17, 331-340 (1974).

5. Friedenstein AJ, Gorskaja JF, Kulagina NN: Fibroblast precursors in normal and irradiated mouse hematopoietic organs. Exp. Hematol. 4, 267-274 (1976).

6. Gronthos S, Zannettino AC, Hay SJ et al.: Molecular and cellular characterisation of highly purified stromal stem cells derived from human bone marrow. J. Cell Sci. 116, 1827-1835 (2003).

7. Friedenstein AJ, Latzinik NV, Gorskaya YF, Luria EA, Moskvina IL: Bone marrow stromal colony formation requires stimulation by haemopoietic cells. Bone Miner. 18, 199-213 (1992).

8. Friedenstein AJ, Chailakhyan RK, Gerasimov UV: Bone marrow osteogenic stem cells: in vitro cultivation and transplantation in diffusion chambers. Cell Tissue Kinet. 20, 263-272 (1987).

9. Owen M, Friedenstein AJ: Stromal stem cells: marrow-derived osteogenic precursors. Ciba Found. Symp. 136, 42-60 (1988).

10. Caplan AI: Mesenchymal stem cells J. Orthop. Res. 9, 641-650 (1991).

-. Landmark paper that coined the term mesenchymal stem cells.

11. Pittenger MF, Mackay AM, Beck SC et al.: Multilineage potential of adult human mesenchymal stem cells. Science 284, 143-147 (1999).

-. Studies on the multilineage potential of MSCs.

12. Tavassoli M, Crosby WH: Transplantation of marrow to extramedullary sites. Science 161, 54-56 (1968).

13. Picinich SC, Mishra PJ, Glod J, Banerjee D: The therapeutic potential of mesenchymal stem cells. Cell- \& tissuebased therapy. Expert Opin. Biol. Ther. 7, 965-973 (2007).

- Interesting review that discusses the therapeutic potential of MSCs.

14. Bianco P, Robey PG, Simmons PJ: Mesenchymal stem cells: revisiting history, concepts, and assays. Cell Stem Cell 2, 313-319 (2008).

- Interesting review that discusses in detail MSC concept, history and assays.
15. Ishii G, Sangai T, Oda T et al.: Bone-marrow-derived myofibroblasts contribute to the cancer-induced stromal reaction. Biochem. Biophys. Res. Commun. 309, 232-240 (2003).

16. Chauhan H, Abraham A, Phillips JR, Pringle JH, Walker RA, Jones JL: There is more than one kind of myofibroblast: analysis of CD34 expression in benign, in situ, and invasive breast lesions. J. Clin. Pathol. 56, 271-276 (2003).

17. Hung SC, Kuo PY, Chang CF, Chen TH, Ho LL: $\alpha$-smooth muscle actin expression and structure integrity in chondrogenesis of human mesenchymal stem cells. Cell Tissue Res. 324, 457-466 (2006).

18. Wang D, Park JS, Chu JS et al: : Proteomic profiling of bone marrow mesenchymal stem cells upon transforming growth factor $\beta 1$ stimulation. J. Biol. Chem. 279, 43725-43734 (2004).

19. Mori L, Bellini A, Stacey MA, Schmidt M, Mattoli S: Fibrocytes contribute to the myofibroblast population in wounded skin and originate from the bone marrow. Exp. Cell Res. 304, 81-90 (2005).

20. Wakitani S, Saito T, Caplan AI: Myogenic cells derived from rat bone marrow mesenchymal stem cells exposed to 5-azacytidine. Muscle Nerve 18, 1417-1426 (1995).

21. Neuhuber B, Gallo G, Howard L, Kostura L, Mackay A, Fischer I: Reevaluation of in vitro differentiation protocols for bone marrow stromal cells: disruption of actin cytoskeleton induces rapid morphological changes and mimics neuronal phenotype. J. Neurosci. Res. 77, 192-204 (2004).

22. Friedman MS, Long MW, Hankenson KD: Osteogenic differentiation of human mesenchymal stem cells is regulated by bone morphogenetic protein-6. J. Cell. Biochem. 98, 538-554 (2006).

23. Gregory CA, Prockop DJ, Spees JL: Non-hematopoietic bone marrow stem cells: molecular control of expansion and differentiation. Exp. Cell Res. 306, 330-335 (2005).

24. Condeelis J, Pollard JW: Macrophages: obligate partners for tumor cell migration, invasion, and metastasis. Cell 124, 263-266 (2006).

- Demonstrated tumor cells and macrophage association. 
25. Mani SA, Guo W, Liao MJ et al.: The epithelial-mesenchymal transition generates cells with properties of stem cells. Cell 133, 704-715 (2008).

-. Demonstrated that cancer cells can generate MSC-like cells by undergoing epithelial-mesenchymal transition (EMT).

26. Hay ED: The mesenchymal cell, its role in the embryo, and the remarkable signaling mechanisms that create it. Dev. Dyn. 233, 706-720 (2005).

27. Perez-Pomares JM, Munoz-Chapuli R: Epithelial-mesenchymal transitions: a mesodermal cell strategy for evolutive innovation in Metazoans. Anat. Rec. 268, 343-351 (2002).

28. Thiery JP, Sleeman JP: Complex networks orchestrate epithelial-mesenchymal transitions. Nat. Rev. Mol. Cell Biol. 7, 131-142 (2006).
- Interesting review on EMT.

29. Yang J, Weinberg RA: Epithelialmesenchymal transition: at the crossroads of development and tumor metastasis. Dev. Cell 14, 818-829 (2008).

- Recent review on EMT.

30. Vernon AE, LaBonne C: Tumor metastasis: a new twist on epithelial-mesenchymal transitions. Curr. Biol. 14, R719-R721 (2004).

31. Jones BJ, McTaggart SJ: Immunosuppression by mesenchymal stromal cells: from culture to clinic. Exp. Hematol. 36, 733-741 (2008).

\section{Website}

101. Raunig G (2004)

www.republicart.net/disc/aap/ raunig07_en.htm

\section{Affiliations}

- Prasun J Mishra

Laboratory of Cancer Biology and Genetics, National Cancer Institute, National Institutes of Health, Bethesda, MD 20892-24264, USA

Tel.: +1 3014518522

Fax: +1 3014807618 mishrapj@mail.nih.gov

- Glenn Merlino Laboratory of Cancer Biology and Genetics, National Cancer Institute, National Institutes of Health, Bethesda, MD 20892-4264, USA

Tel.: +1 3014964270

Fax: +1 3014807618

gmerlino@helix.nih.gov 